\title{
Influence of the probing definition on the flatness measurement
}

\author{
Djezouli Moulai-khatir ${ }^{1, *}$, Eric Pairel $^{2}$, and Hugues Favreliere ${ }^{2}$ \\ ${ }^{1}$ Abou Bekr Belkaid University, Tlemcen, LaRTFM Laboratory, BP 230 Tlemcen, Algeria \\ ${ }^{2}$ Savoie Mont Blanc University, SYMME Laboratory, F-74000 Annecy, France
}

Received: 3 October 2017 / Accepted: 2 October 2018

\begin{abstract}
The quality control of mechanical parts is generally performed on a coordinate measuring machine (CMM). The choice of the number of points to be sampled, their distributions and their positions on the surface, as well as the association criterion remains unresolved. This paper studies the variation of the flatness defect with regard to the number of palpated points. The methodology begins by sampling a cloud of points on a CMM. Then, a modal analysis study is carried out in order to generate a modal surfaces (digitally deformed). Insertion of the coordinate cloud points of these modal surfaces in the GEOVERIF software will allow estimation of the flatness defect. The results of the measured flatness by the three fitting criteria (minimum zone, least squares and minimum volume) are compared.
\end{abstract}

Keywords: CMM / form defect / fitting criterion / probing / flatness

\section{Introduction}

The measurement accuracy of the flatness defect carried out using the coordinate measuring machine (CMM) depends not only on their technical characteristics but also on the strategy used for selecting the number, the location of the points on the surface and the used fitting criterion. The choice of these parameters is left to the initiative of the operator, and the value taken by these parameters has a great influence on the results found.

In the scientific literature, there are many studies that have treated this domain of research. For example, Bourdet [1] proposed a method based on the assumption of small displacement torsors to estimate the gap of flatness, and Kanada and Suzuki [2] developed an optimization method based on nonlinear techniques for the evaluation of the minimum flatness area. Radouani and Anselmetti [3] proposed methods for identifying geometric surfaces based on a solver without the hypothesis of small displacements. Weber et al. [4] introduced a unified linear approximation technique for use in evaluating the forms of straightness, flatness, circularity and cylindricity. Nonlinear equation for each form is linearized using Taylor expansion, then solved as a linear program using software written in $\mathrm{C}++$ language. Huang [5] developed a method to show that straightness and flatness errors can be obtained without the construction of the whole convex hull. Samuel and Shunmugam [6] presented techniques for evaluating circularity and sphericity errors from CMM data. They

\footnotetext{
* Corresponding author: moulai_khatir@yahoo.fr
}

evaluated the form errors directly from CMM data by employing circle/sphere as assessment features and using normal deviations. Gapinski et al. [7] studied the influence of the measuring points number and the type of the roundness deviation on the result (since different fitting elements are used). They gave a recommendation on the measuring points number. Jalid et al. [8] proposed a new method to estimate the form error and the associated uncertainty, which is caused by the measuring process, and analysed the effect of sample size to evaluate the optimum value, which meets the requirement of measurement uncertainty. The majority of the studies were presented by the effect of only one fitting criterion (least squares (LS)) of the flatness defect assessment. They did not propose a clear solution on the number and distribution of points to be palpated for the flatness measurement.

In this paper, we propose to study the variation of the flatness defect with regard to the number of probed points and their distribution on the surface. In addition, a comparison is made between the flatness results estimated by the three association criteria (minimum zone (MZ), least squares and minimum volume). A recommendation on the measuring points number and the criterion to be used will be given.

\section{Criteria for fitting a perfect mathematical surface to a cloud of points}

Minimum zone is the standard criterion that is used for evaluating the form defect of a surface; it is also known as 


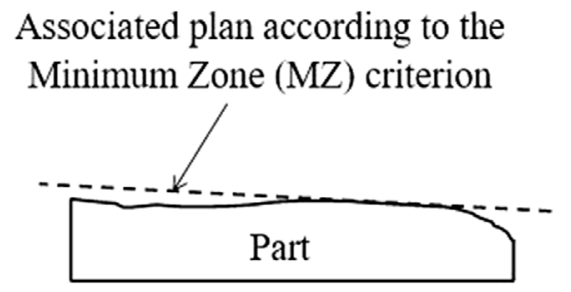

Fig. 1. Substitute feature (MZ) [11].

the Chebyshev criterion. Indeed, for the same distribution of sampled points, this criterion leads to the smallest value, as compared to the other fitting criteria. Nevertheless, this is not the criterion used by default by the software that suggests at least two others:

- the LS criterion;

- the outer or inner matter shell criterion.

With the least squares method, a uniform distribution of points over the entire surface is required [9]. The LS criterion leads to a surface fitted in the "middle" of the sampled points, which means that the points are on the outer side of the matter with respect to the adjusted surface and the others are inside. Some software suggests the option of shifting this adjusted surface to the outermost point, but this action does not change the measured form defect.

The outer or inner matter shell criterion is essentially proposed for palpated circles and cylinders. In this case, it is a matter of maximizing or minimizing the diameter of the circle or the cylinder under the condition that the adjusted surface is on the outside or inside of the matter. This criterion is normalized in order to take the centre of a circle, the axis of a cylinder, or the centre of a sphere as reference.

Regarding the plane, some software packages propose fitting algorithms capable of giving planes adjusted on the external side of matter with respect to the points, so that these planes can again be used as a reference, because as everyone knows, the MZ criterion may lead to fittings that are not representative of the actual surface, as it was illustrated by Pairel [11] on a "ski-shaped" surface, as shown in Figure 1.

Requicha [10] proposed the criterion of the minimum sum of deviations under the condition that the surface is outside the points with respect to matter, and these deviations all have the same sign. Pairel showed that when the area surrounding each point is taken into account, the aforementioned criterion amounts to minimizing the volume between the adjusted surface and the real surface. He generalized the matter outer shell criterion to any surface. This is the reason he called it the minimum volume (Sei) criterion.

The experimental software, GEOVERIF [11-14], which Pairel developed jointly with a computer scientist from the SYMME Laboratory, may implement the above-mentioned criterion as well as the other two criteria, namely, the MZ criterion and the LS criterion.

Once adjusted, the surface that minimizes the form defect rests only on the outermost and innermost points. The question of stability of this criterion arises, a priori,

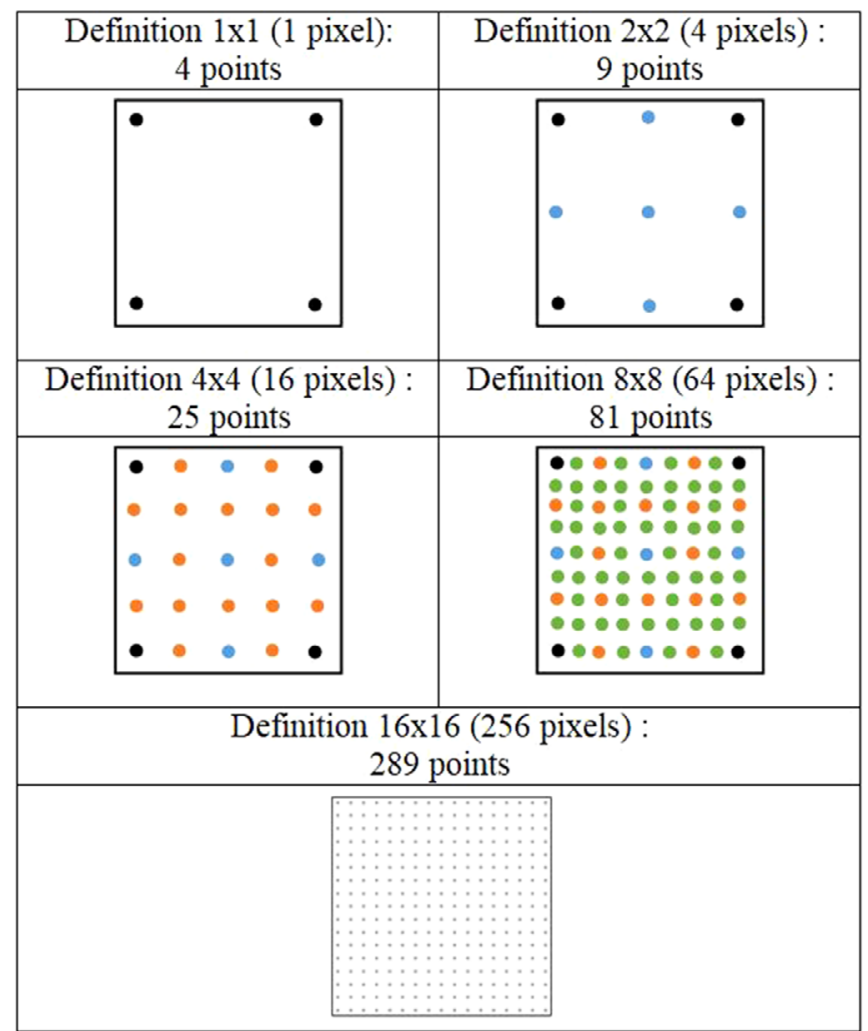

Fig. 2. The five definitions used.

with regard to the distribution of points on the surface. In fact, it is enough to eliminate one of these points of the adjusted surface so that it tilts, possibly strongly, toward the other points of support. This problem seems to be less critical for the other two criteria, i.e., the LS criterion and the Sei criterion, which take into account all the sampled points. They are, a priori, more stable with respect to the distribution of points, even if the minimum sum leads to a surface that rests on the outermost point(s) (but not on the innermost points).

This stability of fitting is therefore studied with respect to the number of points of support for these three criteria and on various numerically distorted planes.

\section{Distribution of sampled points}

We propose to characterize the distribution of points on the surface not only by using a certain number of points but also by a definition in number of pixels (meshes), just like a digital image, which implies a uniform distribution of points on the surface. Therefore, a definition of $1 \times 1$ suggests a single "pixel" delimited by four points at the vertices of a rectangle or a square, depending on the shape of the contour of the scanned surface. Thus, the stability of the fitting criteria was tested on the following five definitions, namely, $1 \times 1,2 \times 2,4 \times 4,8 \times 8$ and $16 \times 16$ (Fig. 2).

The concept of definition, used in this paper for rectangular pixels, could be extended to other patterns. This still remains to be studied further. 

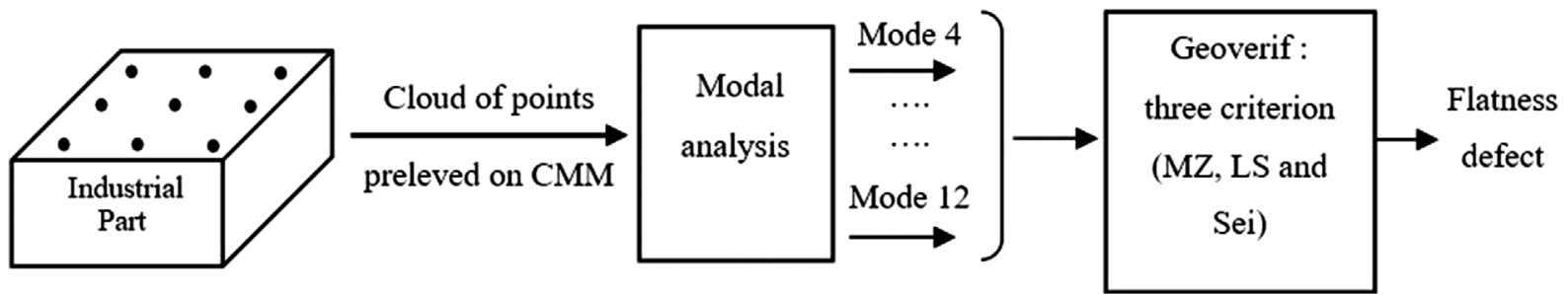

Fig. 3. Methodology for estimating flatness defects according to the number of points.

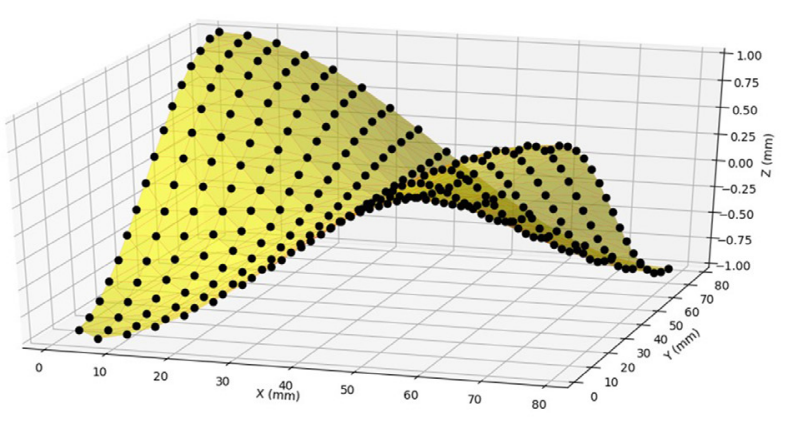

(a)

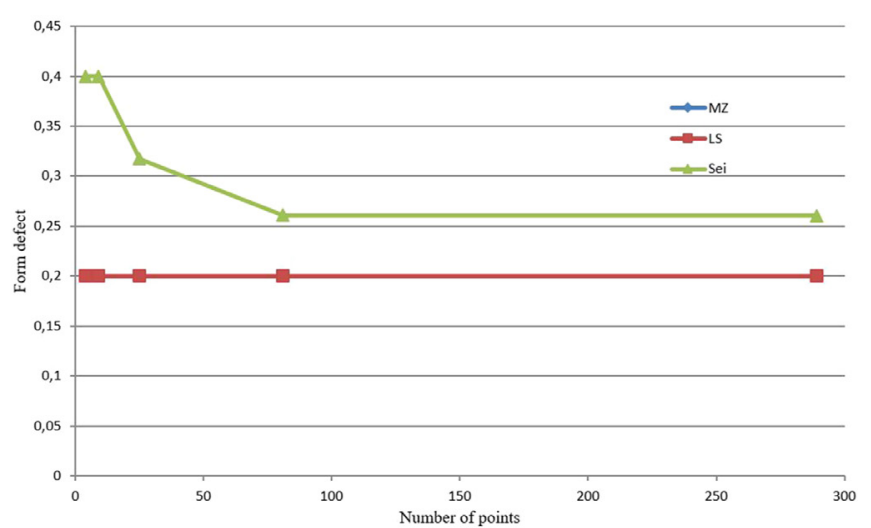

(b)

Fig. 4. Evolution of flatness defect measured according to the three criteria for mode 4 . (a) Mode 4 (saddle of diagonal horse). (b) Variation of the measured flatness defect according to the three criteria.

Figure 3 presents the methodology for estimating flatness defects, followed to conduct this study.

The method begins by sampling a cloud of points, which vary from 4 to 289 points on a CMM. Then, a modal analysis study will be carried out in order to generate a modal surface (digitally deformed) $[15,16]$. Insertion of the coordinates cloud points of these modal surfaces in the GEOVERIF software will allow estimation of the flatness defect.

\section{Deformed planes used to test the influence of the probing definition on the stability of criteria}

In order to test the stability of the fitting criteria on surfaces of various shapes, we generated, by finite elements, the first deformation modes of a thin square plate with a side length of $76 \mathrm{~mm}$, as shown in Figures $4 \mathrm{a}-10 \mathrm{a}$. Indeed, in 2007, these modal forms allowed Samper [15,16] to propose a description language for the form defect. Later, Favreliere applied this language through a "modal" geometrical parameterization [17], in particular to characterize the form defects of a spherical surface [18] and a warped surface [19].

The meshing of the plate corresponds to the definition $16 \times 16$; therefore, each mesh is a square of $4.75 \mathrm{~mm}$ side. The first three modes are rigid modes, i.e., the plate does not deform but only moves. Thus, the first deformation mode is mode 4, which gives a "horse saddle" shape, diagonally with respect to the edges of the surface. The two other modes also have an axisymmetric shape, i.e., showing symmetry relative to a vertical axis passing through the centre of the surface. This has a significant influence on one of the three criteria tested. On the other hand, the higher modes correspond to more "complex" surfaces.

\section{Practical aspects}

The Z-coordinate of the nodes on the various surfaces was divided by 10 in order to have a plausible form defect with regard to their size $\left(76 \times 76 \mathrm{~mm}^{2}\right)$. Then, it was decided to remove one node out of two in order to obtain the definitions $8 \times 8,4 \times 4,2 \times 2$ and finally $1 \times 1$. The coordinates of these nodes were formatted and saved in text files that are understandable by the GEOVERIF software, which was used to calculate the planes adjusted on each definition of each distorted plane, and according to each criterion, this gives $7 \times 5 \times 3=105$ adjustments.

\section{Results and discussion}

Figures $4 \mathrm{a}, 5 \mathrm{a}$ and $6 \mathrm{a}$ show the visualizations of the first three modes (4,5 and 6$)$ and Figures $4 \mathrm{~b}, 5 \mathrm{~b}$ and $6 \mathrm{~b}$ show the evolution of the measured flatness defect according to the three criteria (MZ, LS and Sei) for these three modes. 


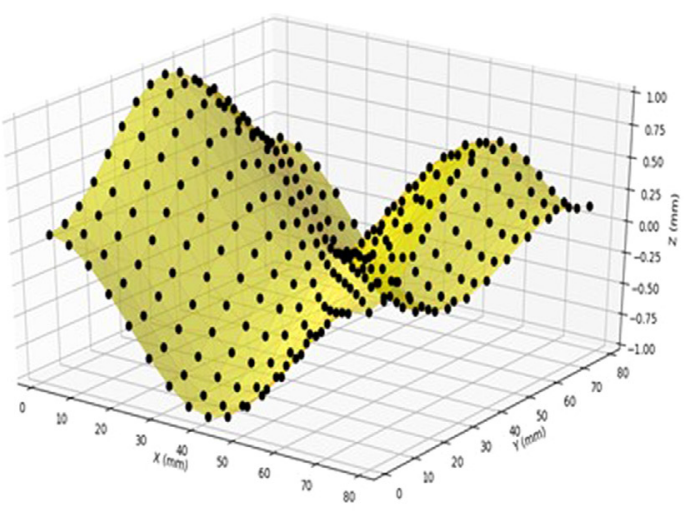

(a)

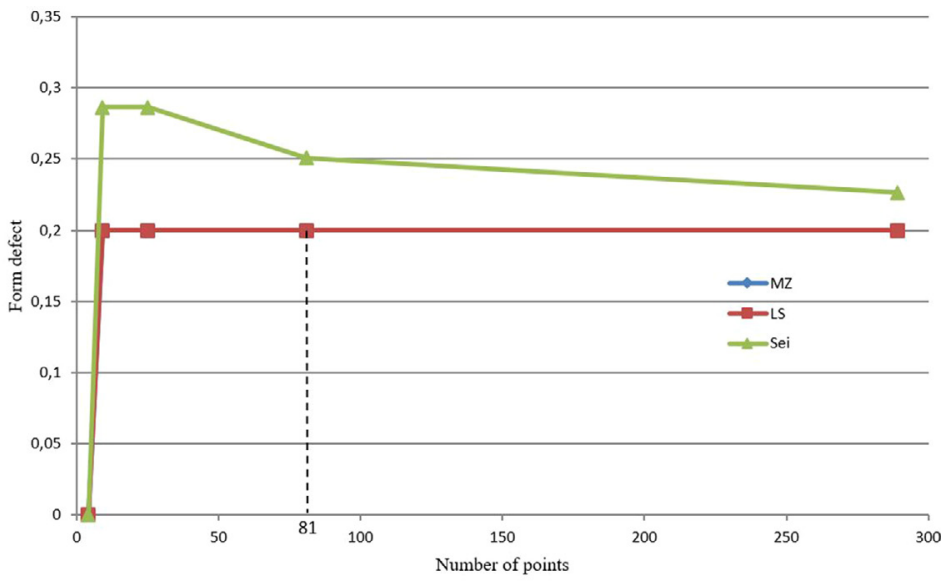

(b)

Fig. 5. Evolution of flatness defect measured according to the three criteria for mode 5. (a) Mode 5 (saddle of parallel horse). (b) Variation of the measured flatness defect according to the three criteria.

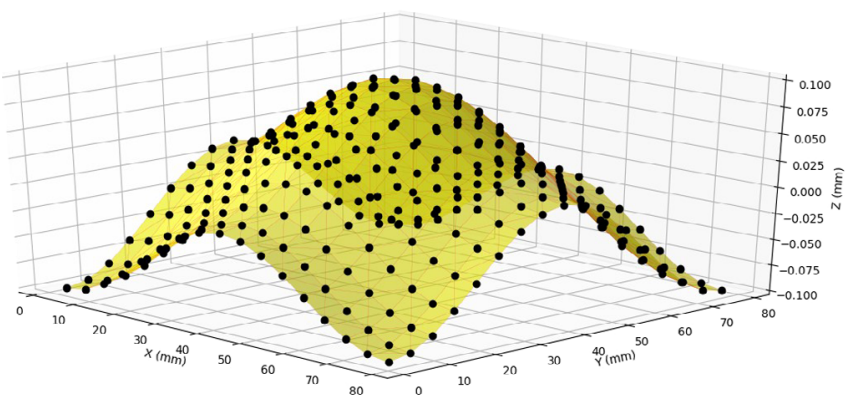

(a)

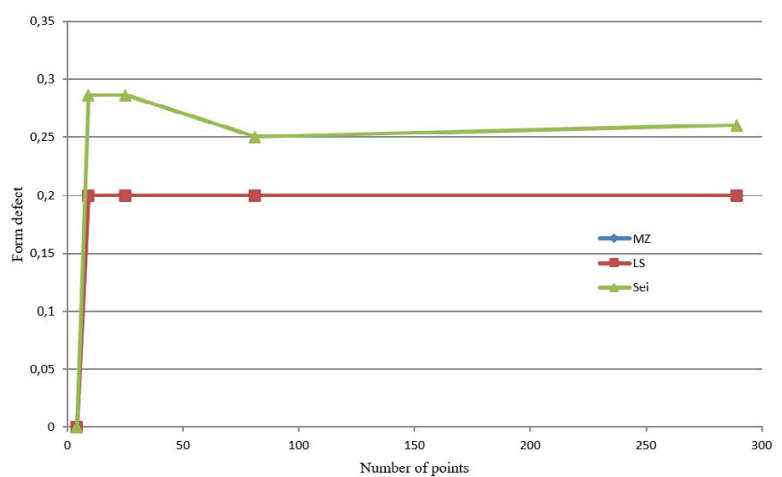

(b)

Fig. 6. Evolution of flatness defect measured according to the three criteria for mode 6. (a) Mode 6 (mono-bump). (b) Variation of the measured flatness defect according to the three criteria.

Figures $7 \mathrm{a}$ and $8 \mathrm{a}$ show the visualizations of the modes ( 7 and 10 ) and Figures $7 \mathrm{~b}$ and $8 \mathrm{~b}$ show the evolution of the measured flatness defect according to the three criteria (MZ, LS and Sei) for these two modes.

Figures $9 \mathrm{a}$ and 10a show the visualizations of the modes (9 and 12) and Figures $9 \mathrm{~b}$ and 10b show the evolution of the measured flatness defect according to the three criteria (MZ, LS and Sei) for these two modes.

Apart from the Sei criterion relative to the first three modes (of axisymmetric form), it is seen that the definition $4 \times 4$ is sufficient for evaluating the form defect. Moreover, the criterion is not very influential. The MZ criterion and the LS criterion are perfectly equivalent on these theoretical forms. This may be explained by the fact that the surface adjusted by these two criteria is always supported on the bumps of these shapes.

The LS criterion gives slightly larger values which can compensate for the underestimated form defect when the other two criteria are used on low definitions $(4 \times 4)$, as it can be seen on the form of mode 7 (diagonal flag - Fig. 7) and the form of mode 10 (parallel flag - Fig. 8).
For modes 9 and 12 (Figs. 9 and 10), the criterion has a little influence. The form defect measured according to the three criteria (MZ, LS and Sei) is practically the same. This is due to the shape (quad-bumps) of these two modes.

The Sei criterion can considerably overestimate the form defect on axisymmetric shapes (modes 4-6 Figs. 4-6), including for high definitions. This is attributed to the fact that an infinite number of planes, bearing on the bump (or bumps) of its axisymmetric surfaces, lead to the same minimum sum of deviations and that the algorithm used by GEOVERIF gives one of the planes bearing on three points; this must result in a maximum deviation almost double that given by the other two criteria. Figure 11 illustrates this situation.

The GEOVERIF algorithm could probably be modified in order to rotate the plane. This is the plane which, among all the planes that minimize the sum of deviations, gives the smallest maximum deviation.

In Table 1, we will present the values of the flatness defects obtained according to the three criteria according to the number of points treated. We have contented 


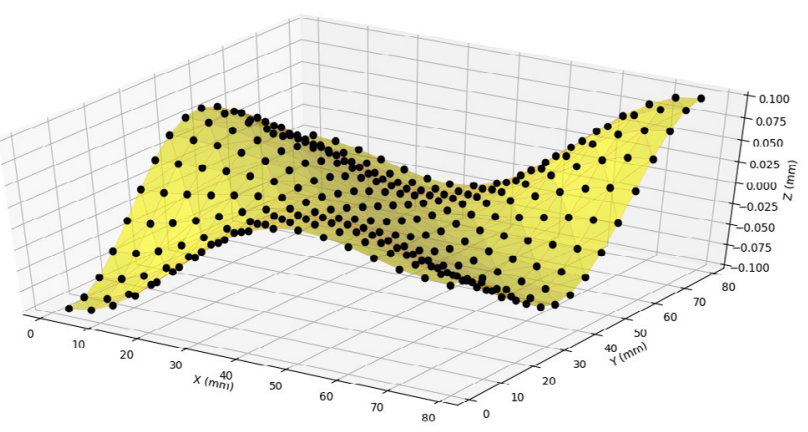

(a)

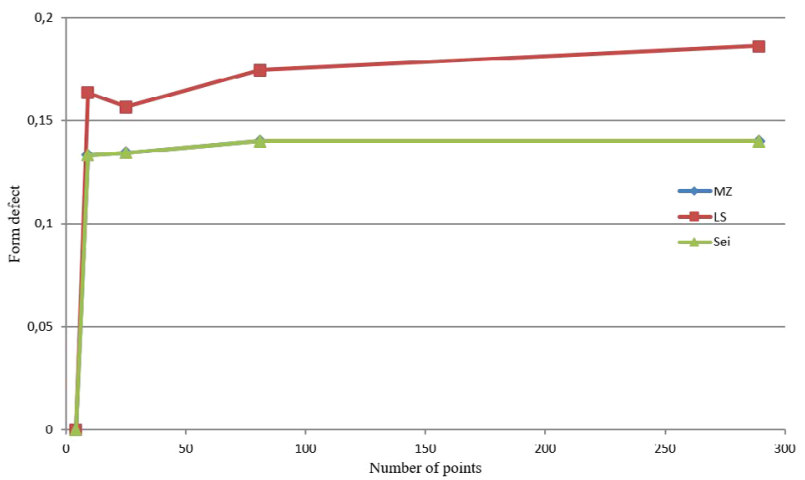

(b)

Fig. 7. Evolution of flatness defect measured according to the three criteria for mode 7. (a) Mode 7 (diagonal flag). (b) Variation of the measured flatness defect according to the three criteria.

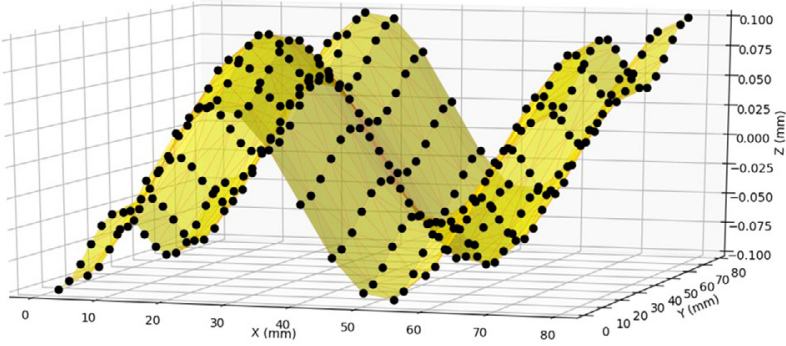

(a)

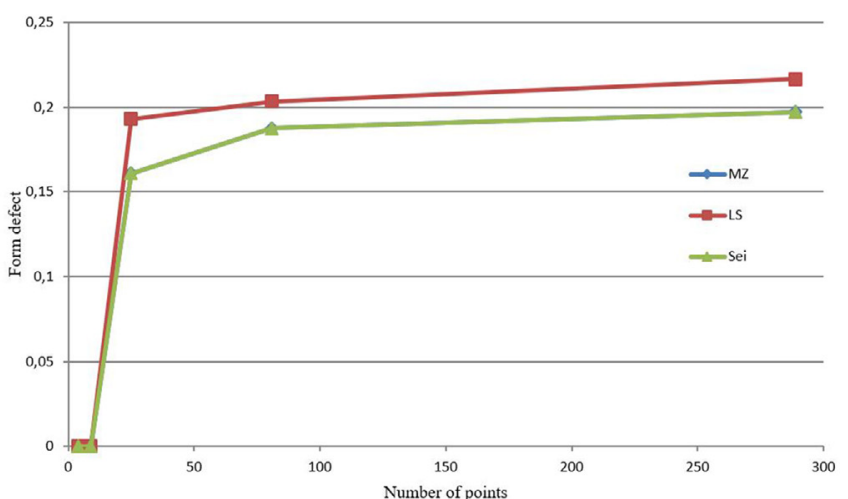

(b)

Fig. 8. Evolution of flatness defect measured according to the three criteria for mode 10. (a) Mode 10 (parallel flag). (b) Variation of the measured flatness defect according to the three criteria.

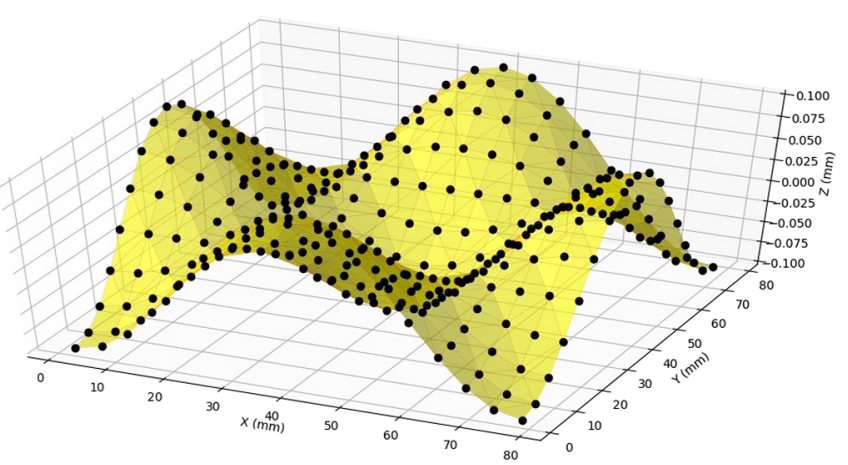

(a)

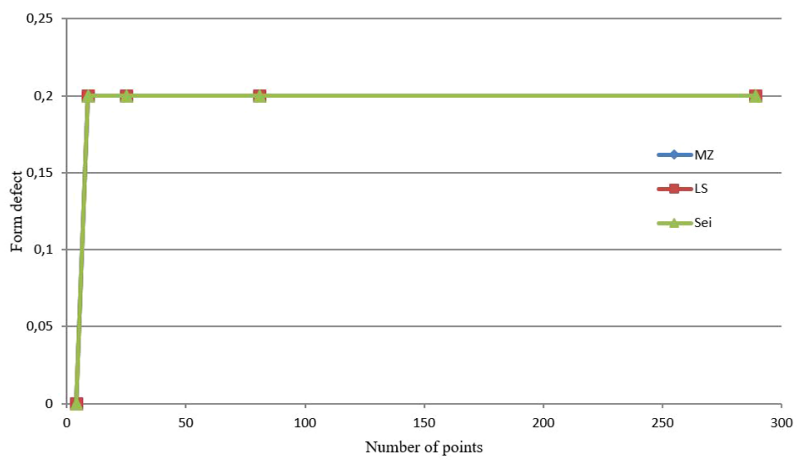

(b)

Fig. 9. Evolution of flatness defect measured according to the three criteria for mode 9. (a) Mode 9 (quad-parallel bumps). (b) Variation of the measured flatness defect according to the three criteria.

ourselves with presenting below only the modes that seem to us the most interesting.

We also note that the MZ criterion gives the value of the smallest form defect. It is in line with the ISO 12781-1:2011 standard [20].

\section{Conclusion}

This study allowed us to show the influence of the probing definition of a surface, on the measured shape defect and according to the three fitting criteria: MZ, LS and Sei. It 


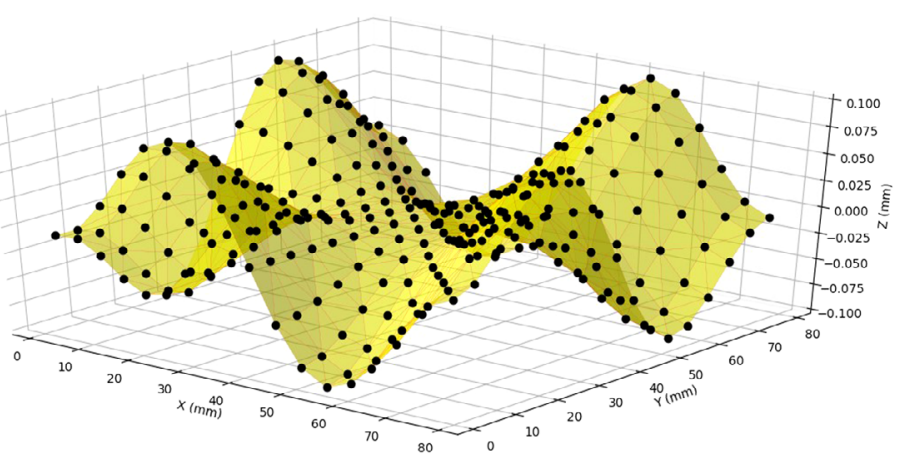

(a)

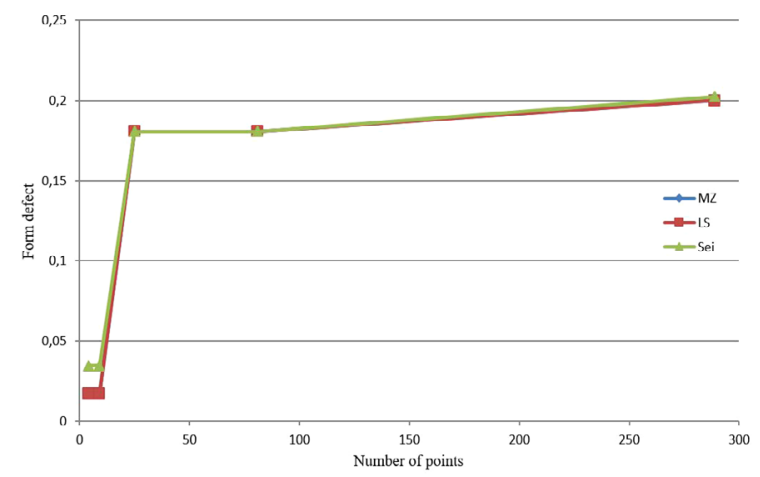

(b)

Fig. 10. Evolution of flatness defect measured according to the three criteria for mode 12. (a) Mode 12 (quad-bumps almost diagonal). (b) Variation of the measured flatness defect according to the three criteria.

Table 1. Values of flatness defects obtained by the three criteria according to the number of points treated.

\begin{tabular}{|c|c|c|c|c|c|c|}
\hline & \multirow{2}{*}{$\begin{array}{l}\text { Flatness defect according } \\
\text { to the criterion }\end{array}$} & \multicolumn{5}{|c|}{ Number of points probed } \\
\hline & & 4 & 9 & 25 & 81 & 289 \\
\hline \multirow[t]{3}{*}{ Mode 5} & $\mathrm{MZ}$ & 0.00001 & 0.199999 & 0.199999 & 0.199999 & 0.199999 \\
\hline & LS & 0.00001 & 0.200001 & 0.200001 & 0.2 & 0.2 \\
\hline & Sei & 0.000019 & 0.28625 & 0.28625 & 0.250698 & 0.226438 \\
\hline \multirow[t]{3}{*}{ Mode 7} & $\mathrm{MZ}$ & 0 & 0.133358 & 0.134475 & 0.139979 & 0.140113 \\
\hline & $\mathrm{LS}$ & 0 & 0.163504 & 0.156709 & 0.174625 & 0.186147 \\
\hline & Sei & 0 & 0.133358 & 0.134475 & 0.139979 & 0.140113 \\
\hline \multirow[t]{3}{*}{ Mode 10} & $\mathrm{MZ}$ & 0.000014 & 0.000036 & 0.160872 & 0.18774 & 0.19713 \\
\hline & $\mathrm{LS}$ & 0.000014 & 0.000039 & 0.19301 & 0.203397 & 0.216512 \\
\hline & Sei & 0.000029 & 0.000043 & 0.160874 & 0.18774 & 0.197137 \\
\hline
\end{tabular}

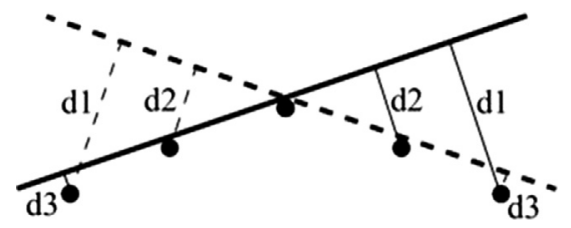

Fig. 11. Over-evaluation of the algorithm used by GEOVERIF of the form defect on the axisymmetric shapes.

shows that, whatever the real surface of the plane, the flatness defect can be evaluated with a rather great precision on a weak probing definition and that the criterion of the least squares seems to be effective and sufficient. It is also shown that the optimal number is defined by 64 pixels, i.e. 81 probing points ( 1 point palpated every $1 / 10$ of the dimension of the piece). This will reduce the measurement time and therefore the cost of control will be minimal. This study could be applied to other forms of surfaces, such as circle, cylinder, cone and sphere. Some surfaces generated by the modal analysis have too much symmetry, but they have the merit to contain only the form defect and thus to have a conclusion extrapolated to the real surfaces. Results of our studies will likely find useful application in automotive and aeronautics industry. Precise and effective easy-to-incorporate methods in surface probing for wings or all external surfaces of fast flying mechanical objects are demanded.

\section{References}

1. P. Bourdet, Contribution à la mesure tridimensionnelle: modèle d'identification géométrique; correction des machines à mesurer; métrologie fonctionnelle des pièces mécaniques, Ph.D. thesis, University of Nancy 1, France, 1988

2. T. Kanada, S. Suzuki, Evaluation of minimum zone flatness by means of nonlinear optimization techniques and its verification, Precis. Eng. 15, 93-99 (1993)

3. M. Radouani, B. Anselmetti, Identification of real surfaces and inspection of the ISO specifications using a solver, Mech. Ind. 4, 249-258 (2003)

4. T. Weber, S. Motavalli, B. Fallahi, S. Hossein Cheraghi, A unified approach to form error evaluation, J. Int. Soc. Precis. Eng. Nanotechnol. 26, 269-278 (2002)

5. J. Huang, An efficient approach for solving the straightness and the flatness problems at large number of data points, Comput. Aided Des. 35, 15-25 (2003) 
6. G.L. Samuel, M.S. Shunmugam, Evaluation of circularity and sphericity from coordinate measurement data, J. Mater. Process. Technol. 139, 90-95 (2003)

7. B. Gapinski, M. Grzelka, M. Rucki, The roundness deviation measurement with coordinate measuring machines, Eng. Rev. 26, 1-6 (2006)

8. A. Jalid, S. Hariri, N.E. Laghzale, Influence of sample size on flatness estimation and uncertainty in three-dimensional measurement, Int. J. Metrol. Qual. Eng. 6, 102 (2015)

9. B. Anselmetti, Manuel de tolérancement: Métrologie avec les normes ISO (Hermes Science Publications, London, 2011), Vol. 5

10. A.A.G. Requicha, Towards a theory of geometrical tolerancing, Int. J. Rob. Res. 2, 4 (1983)

11. E. Pairel, Métrologie fonctionnelle par Calibre virtuel sur Machine à mesurer tridimensionnelle, Ph.D. thesis, University of Savoie, 1995

12. E. Pairel, A new approach for coordinate measurement, in XIV IMEKO World Congress, Tampere, Finland, pp. 278$283(1997)$

13. E. Pairel, Three-dimensional verification of geometric tolerances with the "fitting gauge" model, J. Comput. Inform. Sci. Eng. 7, 1 (2007)
14. E. Pairel, Three-dimensional metrology with the virtual fitting gauges, 11th CIRP International Conference on Computer Aided Tolerancing, France (2009)

15. S. Samper, F. Formosa, Form defects tolerancing by natural modes analysis, J. Comput. Inform. Sci. Eng. 7, 44-51 (2006)

16. F. Formosa, S. Samper, I. Perpoli, Modal expression of form defects, in Models for Computer Aided Tolerancing in Design and Manufacturing, edited by J.K. Davidson (Springer, Dordrecht, The Netherlands, 2007), pp. 13-22

17. H. Favreliere, Modal Tolerancing: From metrology to specifications, Ph.D. thesis, University of Savoie, 2009

18. H. Favreliere, S. Samper, P.A. Adragna, Characterization of defects of a spherical surface by modal decomposition, Int. J. Numer. Eng. Collab. Des. Simul. arXiv:1002.0251v1, (2009)

19. H. Favreliere, S. Samper, P.A. Adragna, M. Giordano, 3D statistical analysis and representation of form error by modal approach, 10th CIRP International Seminar on ComputerAided Tolerancing (2007)

20. Norme ISO 12781-1:2011, Geometrical Product Specifications (GPS) - Flatness - Part 1: Vocabulary and Flatness Parameters (2011)

Cite this article as: Djezouli Moulai-khatir, Eric Pairel, Hugues Favreliere, Influence of the probing definition on the flatness measurement, Int. J. Metrol. Qual. Eng. 9, 15 (2018) 